\title{
First-Principles Molecular-Dynamics Simulations of a Hydrated Electron in Normal and Supercritical Water
}

\author{
Mauro Boero, ${ }^{1,2}$ Michele Parrinello, ${ }^{3}$ Kiyoyuki Terakura, ${ }^{2}$ Tamio Ikeshoji, ${ }^{2}$ and Chee Chin Liew ${ }^{2}$ \\ ${ }^{1}$ Institute of Physics, University of Tsukuba, 1-1-1 Tennodai, Ibaraki 305-8571 Japan \\ ${ }^{2}$ Research Institute for Computational Sciences, National Institute of Advanced Industrial Science and Technology, \\ 1-1-1 Umezono, Tsukuba, Ibaraki 305-8568, Japan \\ ${ }^{3}$ Swiss Center for Scientific Computing (CSCS), via Cantonale, Galleria 2, CH-6928 Manno, Switzerland \\ and Physical Chemistry Department, ETH Hönggerberg HCI, CH-8093 Zurich, Switzerland
}

(Received 14 November 2002; published 6 June 2003)

\begin{abstract}
A first principles study of a hydrated electron in water at ordinary and supercritical conditions is presented. In the first case, the electron cleaves a cavity in the hydrogen bond network in which six $\mathrm{H}_{2} \mathrm{O}$ molecules form the solvation shell. The electron distribution assumes an ellipsoidal shape, and the agreement of the computed and the experimental optical absorption seems to support this picture. At supercritical conditions, instead, the H-bond network is not continuous and allows us to predict that the electron localizes in preexisting cavities in a more isotropic way. Four water molecules form the solvation shell but the localization time shortens significantly.
\end{abstract}

PACS numbers: 71.15.Pd, 32.10.Dk, 61.25.Em

The studies of an excess electron in solution $\left(e_{\mathrm{aq}}^{-}\right)$date back to the early 1960s [1]. Since then, it has been extensively studied experimentally [2-4] and theoretically [5-10] due both to its fundamental importance in physics and to its relevance in a large class of aqueous chemical reactions. Recently, supercritical water above the critical point $(T>647 \mathrm{~K}, P>22.1 \mathrm{MPa})[11,12]$ has been shown to be a promoter of chemical reactions in the absence of acid catalysts [13]. Hence, the excess electron has acquired a new interest also at these extreme thermodynamical conditions. Yet, neither first principles calculations nor experiments have been reported.

In this Letter, we present a Car-Parrinello study of an $e_{\mathrm{aq}}^{-}$in water at both ordinary $(T=300 \mathrm{~K}, \rho=$ $\left.1.00 \mathrm{~g} / \mathrm{cm}^{3}\right)$ and supercritical $(T=653 \mathrm{~K}, \quad \rho=$ $0.73 \mathrm{~g} / \mathrm{cm}^{3}$ ) conditions for which previous simulations $[14,15]$ have already assessed the reliability of our approach. Our aim is to attempt to shed some light in the microscopic structure of the system, an issue of interest in several practical applications.

We performed Car-Parrinello [16] simulations [17] for $\sim 6$ ps on a preequilibrated 32-molecule $\mathrm{H}_{2} \mathrm{O}$ system [18] at ordinary liquid conditions $T=300 \mathrm{~K}, \rho=1.00 \mathrm{~g} / \mathrm{cm}^{3}$ in a cubic box of side $L=9.865 \AA$ [14] and in the supercritical state $T=653 \mathrm{~K}, \rho=0.73 \mathrm{~g} / \mathrm{cm}^{3}, L=10.943 \AA$ [15]. Valence wave functions were expanded in a plane wave basis set with an energy cutoff of $70 \mathrm{Ry}$, and periodic boundary conditions on the simulation cell were applied. The Brillouin zone was sampled at the $\Gamma$ point only and the density functional (DFT) calculations were performed at the Becke-Lee-Yang-Parr [19] gradient corrected level. The core-valence interaction was described by a norm-conserving Troullier-Martins [20] pseudopotential for oxygen and an analytical Car-von Barth one for hydrogen [21]. A fictitious electron mass of 1200 a.u. and an integration step $\Delta t=3.0$ a.u. ensured good control of the conserved quantities. Since one excess electron was introduced and the lowest unoccupied orbital (LUMO) of the neutral system became singly occupied, spin unrestricted calculations were performed. The maximally localized Wannier functions (WFs) and Wannier function centers (WFCs) technique [22] was used to compute dipole moments and to monitor the electronic structure evolution.

We started the calculation with the normal water $\left(n-\mathrm{H}_{2} \mathrm{O}\right)$ system of Ref. [14] equilibrated for 10 ps. Just after the addition of an excess electron $\left(e_{\mathrm{aq}}^{-}\right)$, very little happens. The state corresponding to this extra electron shows a rather diffuse character similar to what has been reported for supercritical water $\left(s c-\mathrm{H}_{2} \mathrm{O}\right)$ [15]. The hydrogen bond ( $\mathrm{H}$ bond) network needs about $1.6 \mathrm{ps}$ to accommodate the additional $e^{-}$. After this simulation time, the delocalized state becomes localized, the $\mathrm{H}$ bonds of the system rearrange, and water molecules reorient in such a way that a cavity forms in the system, as shown in the example of Fig. 1(a). Typically, when a single lobe appears, six water molecules reorient in such a way that all of them point at least one $\mathrm{H}$ toward the electronic cloud, thus forming a solvation shell in agreement with Ref. [10]. Namely, the $e_{\mathrm{aq}}^{-}$replaces the $\mathrm{H}$ bonds lost by those molecules that face the newly formed cavity. By monitoring the trajectory, we noticed that those cavities survive for a period ranging from $\sim 50 \mathrm{fs}$ to a maximum of about $90 \mathrm{fs}$. Those times are of the same order of magnitude of the typical molecular rotation time constants [24], responsible for the H-bond switching. Then the electron density becomes dispersed and a new cavity forms elsewhere. We computed the electronic density of states (DOS) corresponding to distributions of this singly occupied electronic level and the three lowest unoccupied states, that in Ref. [6] were identified as $s$-like and $p$-like orbitals, respectively. The total DOS is shown as a histogram in 

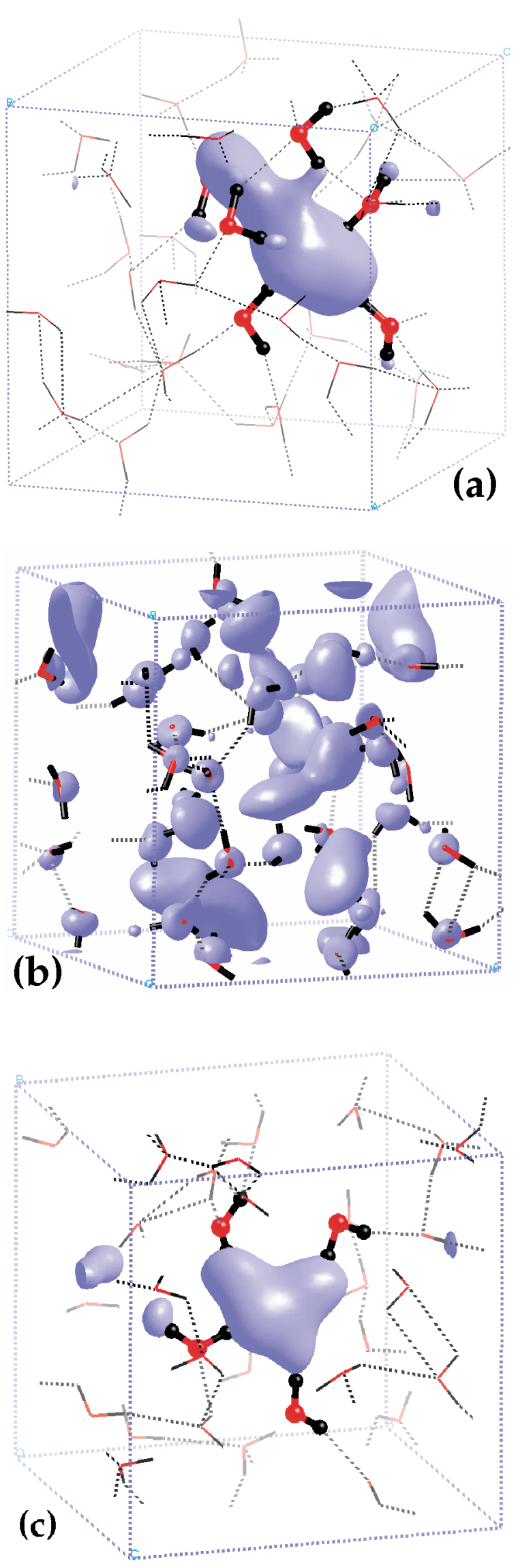

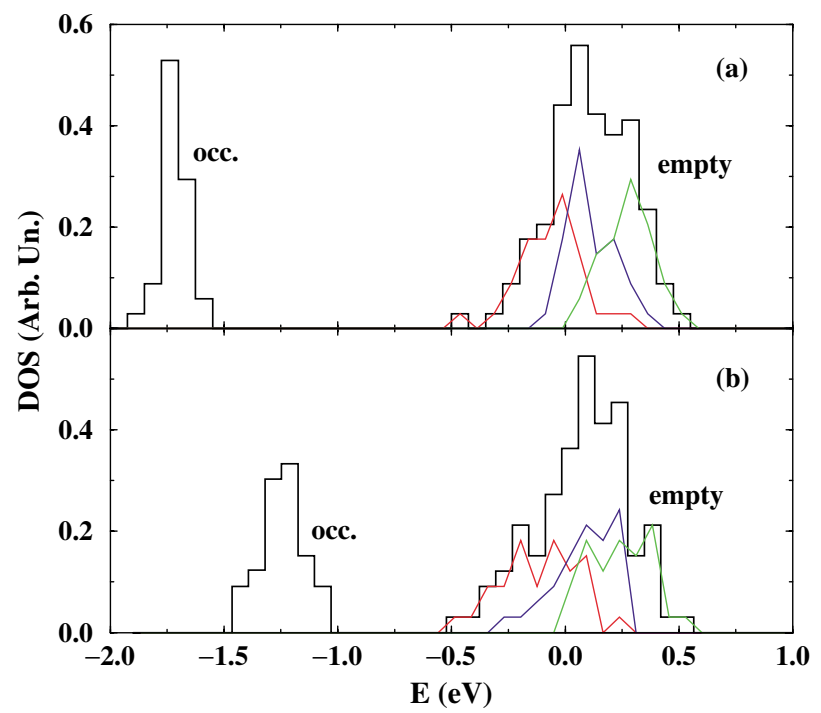

FIG. 2 (color). Density of states (DOS) of the solvated electron (occ.) and the lowest three empty states for $n-\mathrm{H}_{2} \mathrm{O}$ [panel (a)] and $s c-\mathrm{H}_{2} \mathrm{O}$ [panel (b)]. The LUMO, LUMO +1 , and $\mathrm{LUMO}+2$ states are represented by the projected DOS (shown as continuous lines) in red, blue, and green, respectively.

Fig. 2(a). The contributions due to the LUMO, LUMO + 1 , and LUMO + 2 are sketched as red, blue, and green lines, respectively.

By looking at the two distributions corresponding to the LUMO and LUMO + 1, we notice that the two peaks are rather close to each other-separated only by $\sim 0.1 \mathrm{eV}$ on our energy axis - while the maximum of $\mathrm{LUMO}+2$ is more distinct and centered at $0.27 \mathrm{eV}$ above the LUMO +1 peak. We monitored the $e_{\mathrm{aq}}^{-}$density and observed that in a few cases it looks sufficiently isotropic to be described as an $s$-like state. When this occurs, the related three lowest unoccupied states display two pronounced lobes and a node in the cavity that make them similar to $p$-like orbitals. However, other small $\sigma^{*}$ contributions are also present. This makes it difficult to ascribe a definite character to those states. Furthermore, in the majority of the cases, the shape of the $e_{\mathrm{aq}}^{-}$state departs from a pseudospherical distribution. When this occurs, the associated three lowest unoccupied states can lose their $p$-like character and become more diffuse. The lack of a definite angular momentum character increases when the $e_{\mathrm{aq}}^{-}$delocalizes, and this leads to a rather complicated scenario.

In an attempt to make these statements more quantitative, we computed the inertia momenta of the electronic

FIG. 1 (color). Wannier function of the excess electron in $n-\mathrm{H}_{2} \mathrm{O}$ (a) and $s c-\mathrm{H}_{2} \mathrm{O}$ (c). Panel (b) is the $s c-\mathrm{H}_{2} \mathrm{O}$ LUMO state before being occupied. Water molecules of the solvation shell are shown as sticks and balls with the $\mathrm{O}$ in red and the $\mathrm{H}$ in black. Other $\mathrm{H}_{2} \mathrm{O}$ molecules are the thinner sticks. $\mathrm{H}$ bonds are shown as black dashed lines [23]. The isosurfaces (blue) are plotted at a value of $1 \times 10^{-5} \AA^{3}$. 
TABLE I. Average inertia momenta (in $\AA^{2}$ ) and anisotropy factors for the two systems $\left(n-\mathrm{H}_{2} \mathrm{O}\right.$ and $\left.s c-\mathrm{H}_{2} \mathrm{O}\right)$.

\begin{tabular}{cccrc}
\hline \hline System & $J_{1}$ & $J_{2}$ & $J_{3}$ & $W$ \\
\hline$n-\mathrm{H}_{2} \mathrm{O}$ & $6.5 \pm 0.9$ & $7.4 \pm 0.8$ & $12.2 \pm 2.5$ & $0.4 \pm 0.2$ \\
$s c-\mathrm{H}_{2} \mathrm{O}$ & $5.8 \pm 0.9$ & $7.8 \pm 1.1$ & $9.2 \pm 1.8$ & $0.2 \pm 0.2$ \\
\hline \hline
\end{tabular}

distribution by diagonalizing the tensor $J_{\alpha, \beta}$ [25]

$$
J_{\alpha, \beta}=\left\langle\mathbf{r}^{2}\right\rangle \delta_{\alpha, \beta}-\left\langle r_{\alpha} r_{\beta}\right\rangle,
$$

where $\alpha, \beta=x, y, z$. The three computed eigenvalues, averaged on the 6 ps simulation, are reported in Table I, ordered in increasing magnitude, together with an anisotropy factor [26] defined as $W=1-\frac{1}{2}\left(J_{1}+J_{2}\right) / J_{3}$, where $J_{1} \leq J_{2} \leq J_{3}$. In the limiting case of a sphere, it reads $J_{1}=J_{2}=J_{3}, W=0$, while for a line, $J_{1}=J_{2}=0$ and $J_{3} \neq 0, W=1$. Therefore, the average value $W=$ $0.4 \pm 0.2$ for the electron solvated in $n-\mathrm{H}_{2} \mathrm{O}$ provides a clear measure of the anisotropy.

In order to make contact with the experiment, we computed the optical absorption spectrum as

$\sigma(\omega)=\operatorname{const} \frac{1}{\omega} \sum_{i, j}\left(f_{i}-f_{j}\right)\left|\left\langle\psi_{i}|\hat{\mathbf{p}}| \psi_{j}\right\rangle\right|^{2} \delta\left(\epsilon_{i}-\epsilon_{j}-\hbar \omega\right)$,

where $\psi_{i}$ are the Kohn-Sham eigenstates, $\epsilon_{i}$ their corresponding eigenvalues, $f_{i}$ the occupation number, and the difference $f_{i}-f_{j}$ takes care of the fermionic occupancy. The result is reported in Fig. 3(a), where the histogram shows the computed absorption curve, while the dashed curve is the experimental result of Ref. [2]. Experimentally, a peak is detected at $1.7 \mathrm{eV}$ with a dispersion of $0.8 \mathrm{eV}$, while our curve is peaked at $1.74 \mathrm{eV}$ with a dispersion of $0.95 \mathrm{eV}$. On the other hand, previous photoionization experiments agree on a value of $1.2-1.4 \mathrm{eV}$ for the work function at the electrode-water interface [3]. This agreement might well be fortuitous since DFT approaches are well known for the gap underestimation [27]. Nevertheless, the theoretical result is in line with the experimental evidence.

The coordination of water molecules around $e_{\mathrm{aq}}^{-}$was quantified in terms of pair correlation distributions assuming as a reference point the Wannier function center associated to the $e_{\mathrm{aq}}^{-}$and looking at the distances of this WFC from $\mathrm{H}\left(g_{\mathrm{WFC}-\mathrm{H}}\right)$ and $\mathrm{O}\left(g_{\mathrm{WFC}-\mathrm{O}}\right)$ atoms of the surrounding $\mathrm{H}_{2} \mathrm{O}$ molecules. The result is summarized in Fig. 4(a) and accounts for the intuitive picture given in Fig. 1(a), where on average six molecules form the solvation shell of the $e_{\mathrm{aq}}^{-}$.

This same analysis was performed on the $s c-\mathrm{H}_{2} \mathrm{O}$ system, in an attempt to make a prediction, since no experiments have ever been reported. A first consequence of the reduction in the density is the fact that the H-bond network is disrupted and cavities are present [Fig. 1(b)] even before adding the extra electron [15]. Then, after its

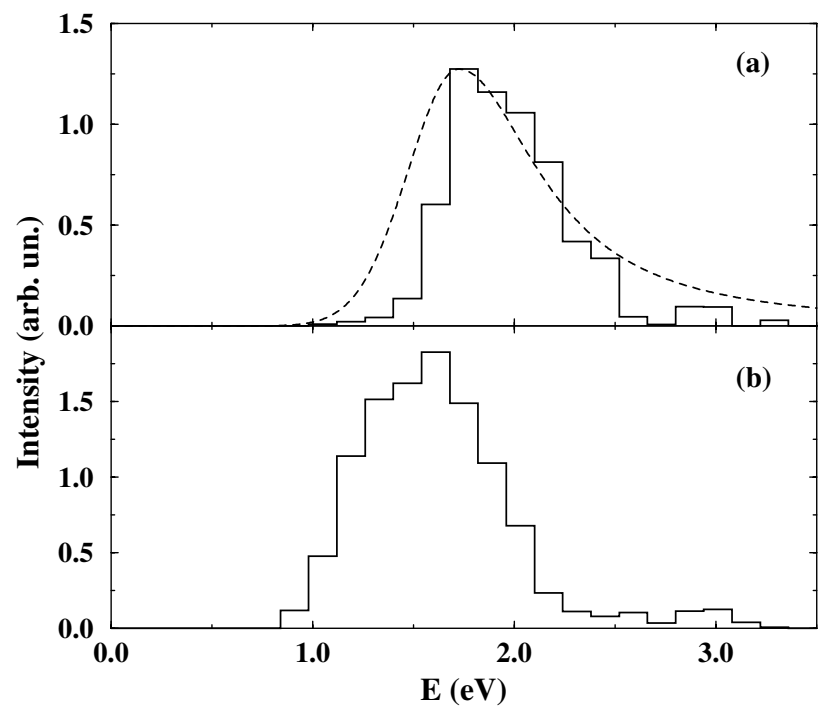

FIG. 3. Optical absorption spectra for the normal liquid (a) and the supercritical system (b). The solid histograms are the computed spectra while in (a); the dashed line represents the experiment of Ref. [2].

introduction, the system reequilibrates and forms a cavity suitable to accommodate the $e_{\mathrm{aq}}^{-}$in about $0.7 \mathrm{ps}$. This time is shorter than the analogous one in $n-\mathrm{H}_{2} \mathrm{O}$. A second consequence is the number of $\mathrm{H}_{2} \mathrm{O}$ molecules that contribute to form the solvation shell. On average only four molecules reorient by pointing one $\mathrm{H}$ toward $e_{\mathrm{aq}}^{-}$as shown

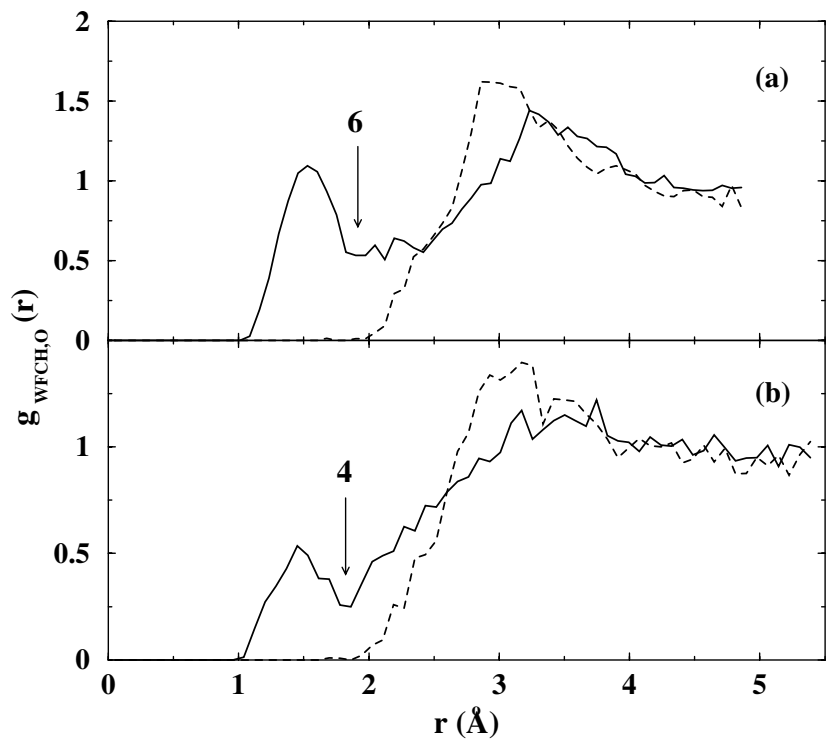

FIG. 4. Pair correlation functions $g_{\mathrm{WFC}-\mathrm{H}}$ (solid line) and $g_{\mathrm{WFC}-\mathrm{O}}$ (dashed line) between the Wannier function center (WFC) of the solvated electron and the $\mathrm{H}$ and $\mathrm{O}$ atoms of the water molecules of the system. The numbers over the arrows indicate the coordination number. Panel (a) refers to $n-\mathrm{H}_{2} \mathrm{O}$, panel (b) to $s c-\mathrm{H}_{2} \mathrm{O}$. The $g_{\mathrm{WFC}-\mathrm{H}}$ and $g_{\mathrm{WFC}-\mathrm{O}}$ in (b) are more noisy because of fluctuations, the statistics being the same. 
in Fig. 1(c). At the same time, the lifetime of the solvation shell is reduced with respect to the ordinary liquid due to the extreme thermodynamical conditions. By looking at the average inertia momenta and the anisotropy factor (Table I), it can be inferred that the more dynamical $\mathrm{H}$ bond network allows for a more isotropical distribution of the electronic charge, hence accounting for the changes in $W(0.2 \pm 0.2)$ with respect to $n-\mathrm{H}_{2} \mathrm{O}$. The DOS relevant to the $e_{\mathrm{aq}}^{-}$is reported in Fig. 2(b). As shown in Ref. [15], the energy level of $e_{\mathrm{aq}}^{-}$approaches the unoccupied states band when the density lowers. As a consequence, the energy gap shrinks and this accounts for the redshift of the absorption curve. In the same figure, the LUMO, $\mathrm{LUMO}+1$, and LUMO +2 peaks are reported as continuous lines as in the case of $n-\mathrm{H}_{2} \mathrm{O}$. It can be noticed that a large broadening and a more uniform distribution characterize the $s c-\mathrm{H}_{2} \mathrm{O}$ system, contrary to the case of $n-\mathrm{H}_{2} \mathrm{O}$. This is reflected in the optical absorption histogram of Fig. 3(b) which shows a broad peak centered at $1.61 \mathrm{eV}$ with a dispersion of $0.9 \mathrm{eV}$ and a more symmetrical shape with respect to $n-\mathrm{H}_{2} \mathrm{O}$.

The pair correlation functions for the $\mathrm{H}$ and $\mathrm{O}$ atoms with respect to the WFC of the excess electron are sketched in Fig. 4(b). As anticipated, after the first peak the coordination number integrates to four water molecules. This reduction in the number of molecules forming the solvation shell can be ascribed to the density reduction only. The high temperature, instead, contributes to the shortening of the lifetime of the cavities and, as a consequence, a fully localized solvated electron can survive for times $\leq 30 \mathrm{fs}$ before delocalizing. The disruption of the $\mathrm{H}$-bond network and its dynamical nature are also responsible for the removal of all or part of the $p$-like character of the three lowest unoccupied states. In fact, for some configurations, even when the $e_{\text {aq }}^{-}$is well localized, only one (LUMO) or two (LUMO and LUMO + 1) states show a definite $p$-like shape, while $\mathrm{LUMO}+1$ or both $\mathrm{LUMO}+1$ and $\mathrm{LUMO}+2$ are delocalized.

The conclusions that can be drawn from our simulations, supported by the rather good agreement with optical absorption data on liquid water, is that, in the ordinary fluid, the spatial distribution of the electronic charge of the $e_{\mathrm{aq}}^{-}$often departs from an isotropical $s$-like shape and both localized lobes and solvation shells dynamically break and reform. This anisotropy is reflected in the shape of the optical absorption curve. In supercritical water, the low density and the rapid breaking and forming of $\mathrm{H}$ bonds allow for a more isotropic distribution of the $e_{\text {aq }}^{-}$ electronic state. However, according to our predictions, a larger broadening and a shift of the main peak to lower frequencies affect the optical absorption spectra. Both the lifetime of the solvation shell and the number of $\mathrm{H}_{2} \mathrm{O}$ molecules forming the shell itself are reduced with respect to the ordinary liquid. In summary, the present study provides a new insight into electron solvation ef- fects, which are fundamental for understanding aqueous solvation reactions.

We thank Pier Luigi Silvestrelli, Michiel Sprik, Daniel Aktah, Alessandro Laio, and Atsushi Oshiyama for insightful discussions. Calculations were performed on the computers of TACC, CSCS, and ISSP.

[1] E. J. Hart and J.W. Boag, J. Am. Chem. Soc. 84, 4090 (1962); E. J. Hart and M. Anbar, The Hydrated Electron (Wiley, New York, 1970).

[2] F.-Y. Jou and G. R. Freeman, J. Phys. Chem. 83, 2383 (1979).

[3] B. Baron et al., J. Chem. Phys. 68, 1997 (1978); D. Grand et al., Chem. Phys. 44, 73 (1979).

[4] P. Han and D. M. Bartles, Phys. Rev. Lett. 64, 1469 (1990).

[5] J. Schnikter and P. J. Rossky, J. Chem. Phys. 86, 3471 (1987).

[6] J. Schnikter et al., Phys. Rev. Lett. 60, 456 (1988).

[7] F. J. Webster et al., Phys. Rev. Lett. 66, 3172 (1991).

[8] R. N. Barnett et al., J. Chem. Phys. 88, 4421 (1988).

[9] M. Sprik et al., J. Stat. Phys. 43, 967 (1986).

[10] K. S. Kim et al., Phys. Rev. Lett. 76, 956 (1996).

[11] P. Postorino et al., J. Chem. Phys. 101, 4123 (1994); A. K. Soper et al., J. Chem. Phys. 106, 247 (1997).

[12] T. Tassaing et al., Europhys. Lett. 42, 265 (1998).

[13] Y. Ikushima et al., Angew. Chem. Int. Ed. 38, 2910 (1999); D. Bröll et al., Angew. Chem. Int. Ed. 38, 2999 (1999).

[14] P. L. Silvestrelli and M. Parrinello, Phys. Rev. Lett. 82, 3308 (1999); P. L. Silvestrelli and M. Parrinello, J. Chem. Phys. 111, 3572 (1999).

[15] M. Boero et al., Phys. Rev. Lett. 85, 3245 (2000); M. Boero et al., J. Chem. Phys. 115, 2219 (2001).

[16] R. Car and M. Parrinello, Phys. Rev. Lett. 55, 2471 (1985).

[17] CPMD code by J. Hutter et al., MPI für Festkörperforschung and IBM Zurich Research Laboratory, 1995-2002.

[18] In normal water simulations of Ref. [14], it was shown that using 32 or 64 molecules does not change the results significantly.

[19] A. D. Becke, Phys. Rev. A 38, 3098 (1988); C. Lee et al., Phys. Rev. B 37, 785 (1988).

[20] N. Troullier and J. L. Martins, Phys. Rev. B 43, 1993 (1991).

[21] See, e.g., M. Sprik et al., J. Chem. Phys. 105, 1142 (1996).

[22] N. Marzari and D. Vanderbilt, Phys. Rev. B 56, 12847 (1997).

[23] The adopted geometrical definition of $\mathrm{H}$-bond is $\mathrm{H}-\mathrm{O}_{\text {acceptor }} \leq 2.50 \AA$, and $\angle \mathrm{O}_{\text {donor }} \mathrm{HO}_{\text {acceptor }} \geq 90^{\circ}$.

[24] G. Gallot et al., J. Chem. Phys. 117, 11301 (2002).

[25] P. L. Silvestrelli et al., Phys. Rev. B 53, 12750 (1996).

[26] See, e.g., T. Ikeshoji, Surf. Rev. Lett. 3, 247 (1996).

[27] We computed the HOMO-LUMO transition energies within the Slater approach for some configurations to estimate the error in the optical gap. The corrections were found to be in the range $0.08-0.05 \mathrm{eV}$. 